\title{
Effect of Prenatal Isotretinoin on Postnatal Development of Cornea and Lens in Albino Wistar Rat: A Morphometric and Histopathlogical Analysis
}

\author{
Divya Premchandran ${ }^{1}$, Sampath Madhyastha ${ }^{2 *}$, Vasudha Saralaya ${ }^{3}$, \\ Teresa Joy ${ }^{4}$, Sudhanshu Sahu ${ }^{5,}$ Rachana $\mathrm{K}^{6}$ \\ ${ }^{I}$ MBBS, MD Anatomy, Kasturba Medical College, Mangalore, Manipal University, India \\ ${ }^{2}$ Additional Professor in Anatomy Kasturba Medical College, Mangalore Manipal University, India - 575001 \\ ${ }^{3} \mathrm{MBBS}, \mathrm{MS}$ Anatomy, HOD, Dept of Anatomy, KMC, Mangalore \\ ${ }^{4} \mathrm{MSc}$ in Anatomy, Lecturer, Dept of Anatomy, KMC, Mangalore \\ ${ }^{5}$ Dept of Biochemistry, KMC, Mangalore \\ ${ }^{6}$ Rachana K: BAMS, Msc Anatomy. KMC, Mangalore
}

\begin{abstract}
Isotretinoin is a known teratogen affecting the neuronal differentiation in developing brain. Apart from its effects on brain and other craniofacial malformations, it is also known to cause failure of detachment of the lens from the surface ectoderm. Interestingly very little is known about the teratogenic effect of isotretinoin on the development of eye. Hence in the present study we evaluated the morphometric and histopathological changes in developing cornea and lens in rats during postnatal development.The pregnant rats received $16 \mathrm{mg} / \mathrm{kg}$ dose of isotretinoin during gestation day 1 to 7 or 12 to 18. The control group received equivalent volume of vegetable oil instead of isotretinoin. The pups were sacrificed on day 7 or 14 or 30 for histopathological and morphometric analysis using haematoxylin and eosin staining.The results of the study clearly demonstrate that prenatal isotretinoin severely affects the development and growth of the cornea in both treatment regimes. There was also a considerable damage to the morphology of the lens.This preliminary animal study clearly demonstrates the teratogenic effect of isotretinoin on developing cornea and lens.
\end{abstract}

KEY WORDS: Developing rat cornea, Developing rat lens, Prenatalisotretinoin, Rat eye development

\section{INTRODUCTION}

Acne is a common complaint of adolescents throughout the world. 13-cis-retinoic acid, isotretinoin is a drug used in the treatment of severe acne. It is a retinoid, derived from vitamin A. Isotretinoin is a synthetic retinoid that inhibits the differentiation of sebaceous glands and corrects the keratinization defects in the follicle. It is also known for its anti-inflammatory activity. It is also being advised for other dermatological diseases such as psoriasis, ichthyosis, dermatological lesions of systemic lupus erythematosus, in the prevention of various types of skin cancers, or even as adjunctive therapy of acute promyelocytic leukemia. There is a growing concern with respect to the teratogenicity of isotretinoin in spite of its wide usage [1]. It is known to cause craniofacial malformations by delaying elevation of the palatine shelf, thereby causing cleft palate [2]. These malformations were attributed to the effect of isotretinoin on the retinoic acid receptors of neural crest cells, by altering the normal transforming growth factor beta (TGF-beta) status [3].

A study by Cook et al demonstrates the teratogenic effect of isotretinoin by preventing the lens detachment and causing corneal opacities in mice [4]. But further studies regarding the postnatal effect on lens was not addressed. The purpose of this study was to ascertain micro-anatomical alteration at three key points of eyedevelopment (postnatal day 7, 14 and 30). Though isotretinoin is a known teratogenic agent, it is still being prescribed without proper physician's guidance. Unplanned pregnancy during isotretinoin treatment can lead to serious complications. Hence in the present study we evaluated the teratogenic effect of isotretinoin on cornea and lens. The objectives of the study also involve comparison ofteratogenic effects at early and late-gestational treatment. 


\subsection{Animals and housing conditions}

\section{METHODLOOGY}

In-house bred male and female albino Wistar rats (3-4 months old and weight 200-230g) were selected for the study. Breeding and maintenance of the animals was done as per the guidelines of Government of India for use of Laboratory animals as published in Ind. Journal of Pharmacology (31:92-95, 1999). Institutional Animal Ethics Committee approval was obtained before the conduct of the study (IAEC letter dated 09/05/2012).

\subsection{Mating of rats and animal groups}

Female rats were allowed to mate with fertile sexually active male rats (proportion of two females for every male rat). After 48 hours, vaginal smears were taken in order to verify the presence of sperms and confirm pregnancy. The rats were then designated as day 0 of pregnancy and were housed individually. All dams were allowed to undergo normal delivery. The litter size, body weight and crown-rump lengths were recorded in each group andthe pups were with their biological mothers. Four pregnant rats were used in each group. From each mother one male and one female pup were sacrificed with a high dose of ether and used for postnatal studies on day 7. Hence a total number of 8 pups for each group were considered for the entire studies. The remaining pups were kept with their biological mothers. At postnatal day 14 another set of onemale and one female pups from each group was sacrificed for histopathological studies $(n=8)$. The remaining pups of the litter were again left in their cages with their biological motherstill weaning (21 days after birth) and there after sacrificed on day 30 $(\mathrm{n}=8)$.

Group 1: Control - Pups belonging to pregnant rats who received an equivalent volume of vegetable oil instead of isotretinoin.

Group 2: Pups belonging to pregnant rats who received isotretinoin $(16 \mathrm{mg} / \mathrm{kg}$ body weight dose) during, gestational period 1 to 7 (P, 1-7).

Group 3: Pups belonging to pregnant rats who received isotretinoin $(16 \mathrm{mg} / \mathrm{kg}$ body weight dose) during,gestational period 12 to $18(\mathrm{P}, 12-18)$.

Isotretinoin was administered orally using a metallic oropharyngeal cannula. The human dose of the isotretinoin was converted to the rat dose. In our earlier study $16 \mathrm{mg} / \mathrm{kg}$ dose had proved to be teratogenic on the developing brain [5] hence it was decided to use the same dosage in order to avoid using unnecessary number of animals.

\subsection{Histopathological studies}

On $7^{\text {th }}, 14^{\text {th }}$ and $30^{\text {th }}$ postnatal day, pups were sacrificed for histological studies. Each rat was deeply anaesthetized with ether and followed by trans-cardiac perfusion (with $0.9 \%$ saline and $10 \%$ formalin). The rat was decapitated and the eye globes were removed and kept in $10 \%$ formalin for $48 \mathrm{~h}$. Paraffin blocks were made and sections (either transverse or sagittal) of 3-5- $\mu \mathrm{m}$ thickness were cut using a rotary microtome at the level of optic disc.Twelve sections from each animal were mounted serially on air dried gelatinized slides. The sections were stained with haematoxylin and eosin. In each section of eye, the thickness of the cornea at the center point was measured using ocular micrometer. The percentage of growth of corneal thickness was also evaluated. A qualitative evaluation of corneal epithelium, arrangement of the stromal fibres, structure of descement membrane and endothelial layers were made.

In each section the morphology of the lens, including anterior lens capsule, cortical and nuclear lens fibres and posterior lens capsule were observed.

\section{STATISTICAL ANALYSIS}

The thickness of the corneal values was expressed as mean \pm SD. The significance of differences among the groups was assessed using one way analysis of Variance (ANOVA) test followed by Bonferroni's multiple comparison test. $\mathrm{P}$ values $<0.05$ were considered as significant. 


\section{RESULTS}

There was a highly significant $(P<.001)$ decline in the litter size among both the treatment regimes as compared to the control. Though there was a decline in the birth weight at P, 12-18, the weight gain improved by postnatal day 7 . Subsequently there was no statistically significant difference in the body weight at each point of time weighed. There was no significant difference in CR length among the groups.

At postnatal day 7, the mean thickness of the cornea differed significantly $(P<.001)$ between control and both treatment regimes. The $\mathrm{P}, 12-18$ regimeshowed a significant difference $(P<.05)$ when compared to the $\mathrm{P}, 1-7$ regimen.

Evaluation of corneal thickness at postnatal day 14 and 30 also showed a highly significant decrease for both treatment regimes. Interestingly the comparison of corneal thickness between P, 1-7 with P,12-18 regime did not show any statistically significant difference on both postnatal day 14 as well as day 30 (Fig.1).

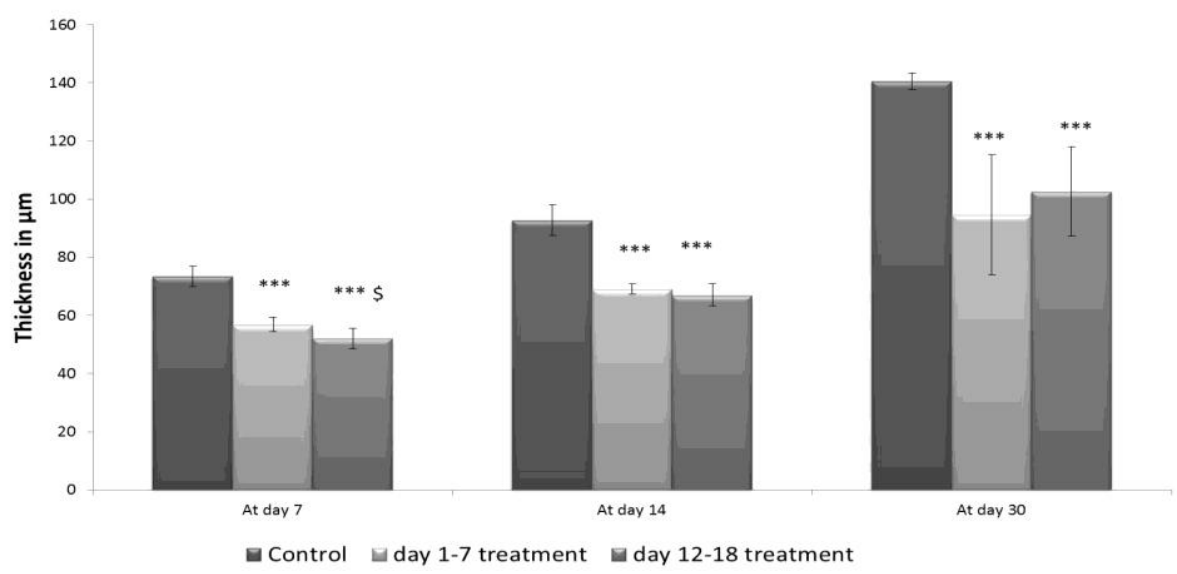

Fig 1 Thickness $(\mu \mathrm{m})$ of the rat cornea measured at the central region at different postnatal days of development $(n=8)$.Values expressed as mean \& the error bar indicates \pm SD. Comparison between control and treated groups. $* * *=P<.001$, comparison between treated groups $\$=P<.05$

Percentage comparison: The growth pattern of thickness in the rat retinal cornea between postnatal day 7 and 14 (at 7 days of growth) was $25.85 \%$ in control group. Similar comparison on day 1-7 of prenatal treatment was $21.54 \%$ and $28.69 \%$ with day $12-18$. The growth pattern between days 14 to 30 (16 days of growth) in control group was $52 \%$, in day $1-7$ of treatment, $36.71 \%$ and in day $12-18$ was $53.22 \%$.

\subsection{Histopathological evaluation of cornea}

In the control group of pups,histopathological evaluation of cornea showed a normal corneal epithelium, regular arrangement of the collagen fibres with numerous fibroblasts on postnatal day 7 (Fig.2).

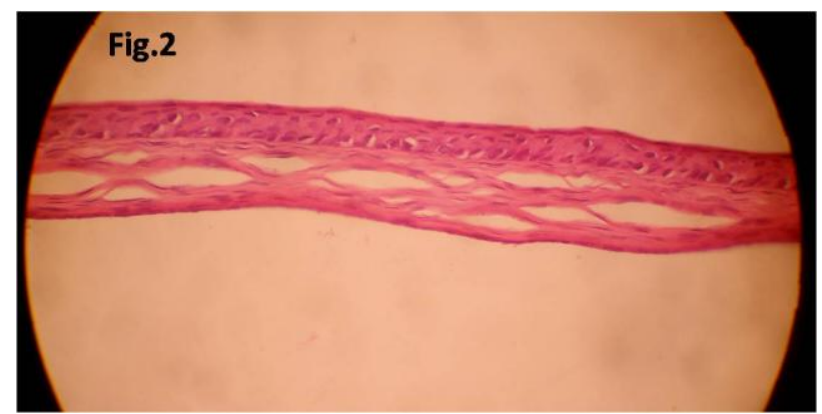

Fig 2Histomicrographic picture showing normal corneal features in control group of rats on postnatal day 7 (Haematoxylin and Eosin staining, under $40 \mathrm{X}$ magnification).

The descement membrane and a single layer of corneal epithelium were evident amongst other normal features on postnatal day 30 .

Histopathological evaluation in both treatment regimes showed an irregular arrangement of stromal fibres in the cornea (Fig.3). 


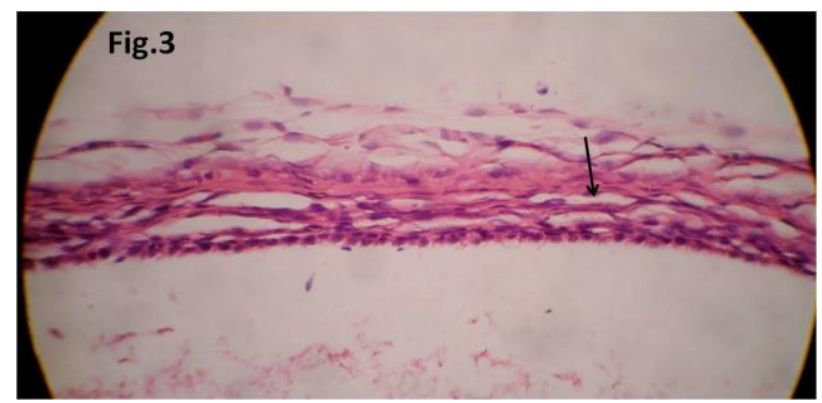

Fig 3 Histomicrographic picture showing irregular arrangement of the stromal fibres in rat cornea on postnatal day 7 at $\mathrm{P}, 1-7$ regime ( $\mathrm{H}$ and $\mathrm{E}$, under $40 \mathrm{X})$.

There was an increase in corneal stromal mitotic activity, characterized by increased fibroblast cell nuclei in the substantiapropria, which was observed in few slides among the treated groups, but this feature was not consistent with all the groups tested (Fig.4).

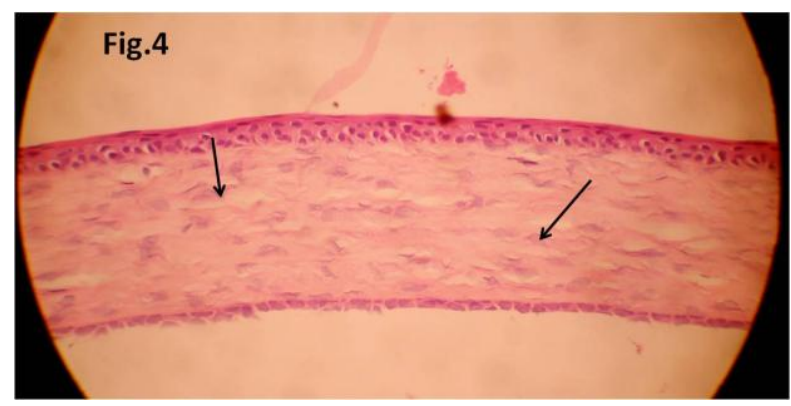

Fig 4Histomicrographic picture showing enhanced stromal mitotic activity characterized by more fibroblast cell nuclei in substantiapropria of rat cornea on postnatal day 7 at $\mathrm{P}, 1-7$ regime ( $\mathrm{H}$ and $\mathrm{E}$ staining, under $40 \mathrm{X}$ ).

Slides of P, 12-18 regime showedafew focal morphological changes and corneal swellings with irregular and widely separated wastage of stromal fibres when examined on day 14 (Fig.5).

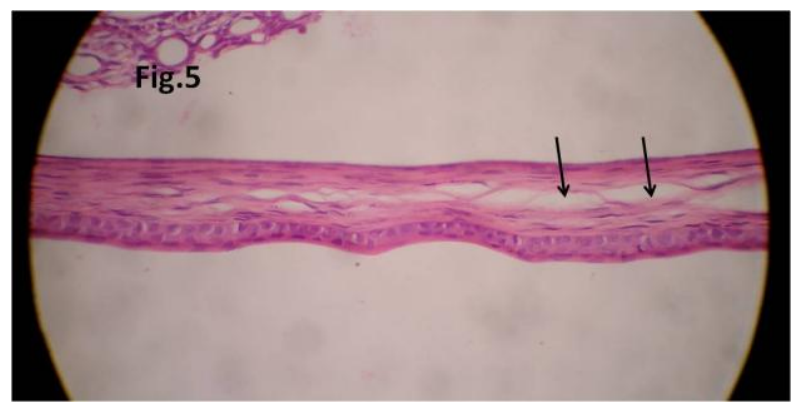

Fig 5Histomicrographic picture showing corneal swellings with irregular and widely separated wastage of sromalfibres of rat cornea on postnatal day 14 at $\mathrm{P}, 12-18$ regime ( $\mathrm{H}$ and $\mathrm{E}$, under $40 \mathrm{X})$.

Corneal endothelial cells agenesis and disruption was evident in treated group when examined at day 7 , 14 and 30. These features are almost consistent with all the treated groups. In addition irregularly oriented, vacuolated and polymorphic endothelial cells were also observed(Fig.6). 


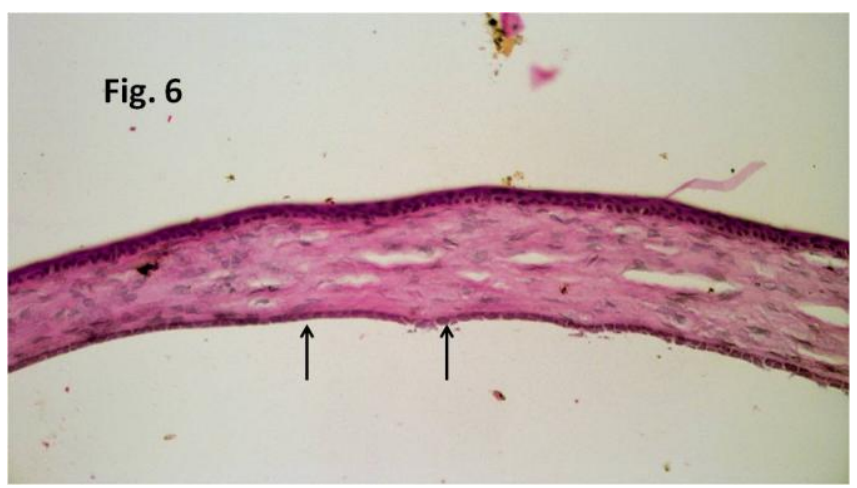

Fig6Histomicrographic picture showing disrupted endothelial cells of the rat cornea ( $\mathrm{H}$ and E, under $20 \mathrm{X})$.

\subsection{Histopathological evaluation of lens}

The lens belonging to the control group showed normal findings with single layer of anterior cuboidal epithelial cells, regular arrangement of cortical and nuclear lens fibres. The posterior lens capsule was clear, without any epithelial cells behind the equator (Fig.7).

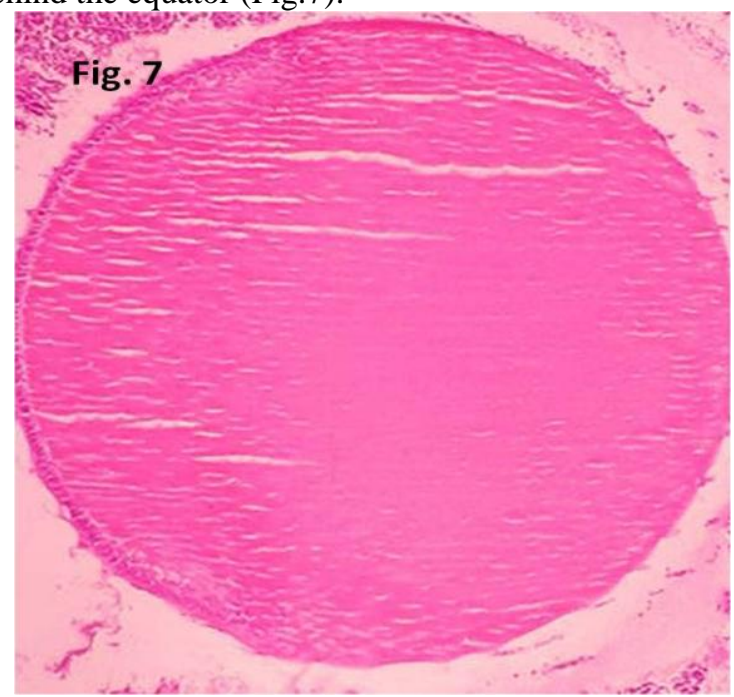

Fig 7Histomicrographic picture showing normal lens with a single layer of anterior cuboidal epithelium and regular arrangement of the lens fibres ( $\mathrm{H}$ and $\mathrm{E}$ staining, under $40 \mathrm{X}$ ).

Prenatal isotretinoin in both treatment regime employed in the study demonstrated a severe damage of the lens structure. The most common finding was irregularly oriented lens fibres. The fibres were swollen at different parts. Prenatal isotretinoin treatment on day 12-18 also showed retention of nuclei (basophilic appearance stained with haematoxylin) in the lens fibres especially behind the equator (Fig.8).

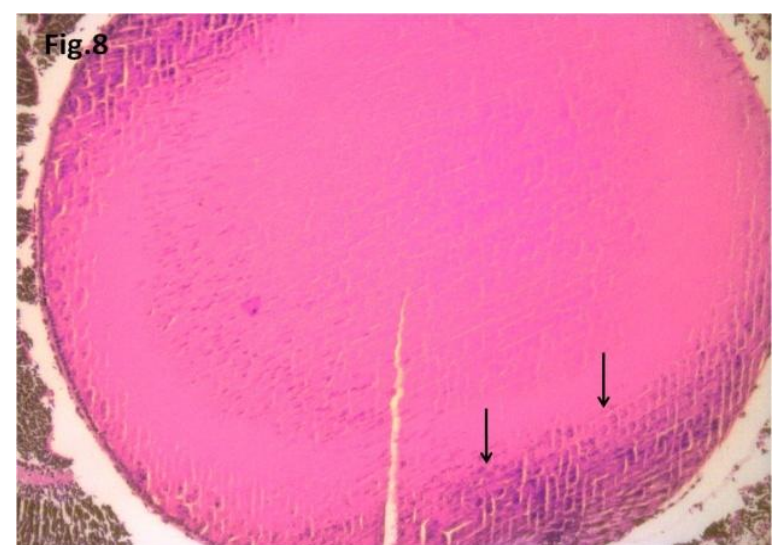

Fig 8Histomicrographic picture showing damaged lens (H and E staining, under $40 \mathrm{X}$ ). 


\section{DISCUSSION}

Lens vesicle detachment is known to occur on day 11 in mice [6] and day 35 in the humans. During normal lens development cells of the posterior wall begin to elongate anteriorly and form long fibres that gradually fill the lumen of the vesicle. In human embryos, by the end of the seventh week, the primary lens fibres reach the anterior wall of the lens vesicle. Secondary lens fibres are continuously added to the central core. During this process of anterior migration, their nuclei normally disintegrate so that the central lens area lacks nuclei. In the present study in few slides belonging to the P, 12-18 regime, exhibited numerous nuclei in the posterior segment of the lens. Congenital cataract occurring in rubella syndrome is characterized by persistent appearance of the lens epithelial nuclei within the lens fibres [7]. By correlating these findings, it can be confirmed that lens programming may be damaged by toxic insult of isotretinoin if a mother consumes it during pregnancy. In histological examination, the lens has an anterior subcapsular single layer of epithelial cells that normally terminates at the lens equator in HandE staining preparation [8]. The posterior lens capsule does not have this layer and appears smooth and thin. The migration of the lens epithelial cells over the posterior lens capsule behind the equator is responsible for the formation of posterior subcapsular cataract [9]. In the present study prenatal isotretinoin treatment during day 12-18 showed similar findings in many rats. Degeneration of corticallensfibres can result in cortical cataract formation [10]. In the present study we also observed degeneration of lens fibres, their irregular arrangement and also swollen fibres at certain area of lens. These observations were consistent with the histopathological findings of cortical cataract.

A study by Cook and Sulik [4] demonstrated a delay in lens detachment on gestation day 17, when isotretinoin was administered prenatally on gestation day 7 . Though the present study does not involve the observation of lens detachment, its postnatal growth and structure was assessed. In the present study gestational day 12-18 treatment proved to be a sensitive period for teratogenic effect on lens on day 7 but not on day 14 or 30 . Hence from our findings it cannot be concluded whether early (1-7) or late (12-18) gestation treatment is more sensitive in inducing teratogenic effect on lens.

Vitamin A is an essential dietary nutrient and precursor of retinoic acid. Retinoic acid (RA) signaling plays multiple roles during embryonic eye development. Cveklrevealed the importance of RA signaling for reciprocal interactions between the invaginating lens placode and the optic cup vesicle.It also promotes the normal development of the retina and the opic nerve through its activities in the neural crest cell-derived periocular mesenchyme [11]. Though retinoic acid is required for normal development of eye and many other structures, it is proven to be teratogenic at a higher dose and during a sensitive period.

The corneal epithelial layer is derived from the surface ectoderm; the mesenchymal tissue in front of the developing eye forms the substantiapropria.Abnormal lens detachment can result in mechanical interference with neural crest migration (between the surface ectoderm and the lens)which is destined to form the corneal stroma and endothelium, and even stroma of the iris ${ }^{[4]}$ Hence in the present study the histopathological changes observed in the cornea may be due to defective migration of neural crest cells, which is a consequence of delayed lens detachment. An immunohistochemical method labeling the neural crest cells in cornea and lens during, different points of prenatal and postnatal development would provide further insight into isotretinoin induced morphological defects in lens and cornea.

\section{CONCLUSION}

Prenatal isotertinoin treatment in rats during any gestation period is known to deform the lens structure as well as cornea.

\section{REFERENCES}

[1] Berarda A, Azoulay L, Koren G, Blais L, Perrealt S \&Oraichi D. Isotretinoin, pregnancies, abortions and birth defects: a population-based perspective. Br J ClinPharmacol63, 2007, 196-205.

[2] Eleny BR, Karina GS, Maria AJ. Effect of isotretinoin on tooth germ and palate development in mouse embryos. Braz. Dent. J, 12,2001,115-19.

[3] O'Reilly KC, Shumake J, Gonzalez-Lima F, Lane MA, Bailey SJ. Chronic administration of 13-Cis-Retinoic Acid Increases Depression-Related Behavior in Mice. Neuropsychopharmacology, 31, 2006,1919-27.

[4] Cook CS and Sulik K.K. Laminin and fibronectin in Retinoid-induced keratolenticulardysgnesis. Investigative ophthalmology and Visual Science, 31, 1990,751-57.

[5] Divya P, Sampath M, V, Jai A, Teresa J, Sudhanshu S. Effect of Prenatal Isotretinoin Exposure on Neuronal Population of Prefrontal Cortex in Rats. Research Journal of Pharmaceutical, Biological and Chemical Sciences, 4, $2013,902-11$.

[6] Cook CS and Sulik KK. Sequential scanning electron microscopic analyses of normal and spontaneously occurring abnormal ocular development in C57BI/6J mice. Scanning Electron Microsc, 3, 1986,1215

[7] Klintworth GK and Garner A editors. The causes, types and morphology of cataract.In Pathology of ocular disease: a Dynamic Approach. 2nd ed. New York: Marcel Dekker 1994.

[8] Doganay S, Turkoz Y, Evereklioglu C, BorazanM and Ozerol E. Use of caffeicacidphenethyl ester to prevent sodium seleniteinduced cataract in rat eyes. J Cataract Refract Surg, 28, 2002,1457-62.

[9] Evereklioglu C, Ozkiris A, Alasehirli B, Sari I, Guldur E, Cengiz B et al., Effect of gestational nicotine treatment on newborn rat retina: a histopathological and morphometric analysis. Ophthalmic Physiol Optics, 23, 2003, 527-33.

[10] Klintworth GK and Eagle RC Jr editors. The eye and ocular adnexa. In: Stephen SS. Diagnostic Surgical Pathology.3rd ed.Philadelphia: Lippincott, Williams and Wilkins 1999.

[11] Cvekl A and Wang WL. Retinoic acid signaling in mammalian eye development. Exp Eye Res, 89, 2009,280-91. 